\title{
Leaps: an approach to the block structure of a graph
}

\author{
Henry Martyn Mulder \\ Econometrisch Instituut, Erasmus Universiteit \\ P.O. Box 1738, 3000 DR Rotterdam, The Netherlands \\ e-mail: hmmulder@few.eur.nl \\ Ladislav Nebeský * \\ Filozofická fakulta, Univerzita Karlova v Praze \\ nám. J. Palacha 2, 11638 Praha 1, Czech Republic \\ e-mail: Ladislav.Nebesky@ff.cuni.cz
}

ECONOMETRIC Institute Report EI 2004-49

\begin{abstract}
To study the block structure of a connected graph $G=(V, E)$, we introduce two algebraic approaches that reflect this structure: a binary operation + called a leap operation and a ternary relation $L$ called a leap system, both on a finite, nonempty set $V$. These algebraic structures are easily studied by considering their underlying graphs, which turn out to be block graphs. Conversely, we define the operation $+_{G}$ as well as the set of leaps $L_{G}$ of the connected graph $G$. The underlying graph of $+_{G}$, as well as that of $L_{G}$, turns out to be just the block closure of $G$ (i.e. the graph obtained by making each block of $G$ into a complete subgraph).
\end{abstract}

*supported by SWON, Ministry of Education and Science, The Netherlands 


\section{Introduction}

An important aspect of a connected graph $G$ is the structure of its blocks and cutvertices. Recall that a block in a graph is a maximal connected nonseparable subgraph, where nonseparable means without cut-vertices. A bridge with its ends is called a trivial block. There exist various ways to study this block structure. The classical one is probably the intersection graph $\mathcal{H}(G)$ of the blocks of $G$, see $[2,3]$. This intersection graph is called the block graph of $G$. It is easily seen that all blocks in $\mathcal{H}(G)$ are complete. This is the origin of the term block graph being a connected graph, in which all blocks are complete. Another recent approach was taken in [1] involving the all-paths transit function of a graph. A transit function is a function on a graph that returns a nonempty set for each pair of vertices. The all-paths transit function returns, for the pair $u, v$, the set of vertices on the $u, v$-paths.

In this paper we propose two different algebraic methods to study the block structure: a binary operation called leap operation and a ternary relation called leap system. Both methods involve the idea of leaping from vertex to vertex, instead of walking along edges. Although there are differences between the two approaches, they are closely related. Therefore, in both cases, we use the same term 'leap'. They are based on two different characterizing properties of blocks. The underlying idea for the leap operation is the following. Let $u$ and $w$ be two vertices in $G$. Now we want to know which vertices can be avoided by any $u, w$-walk $P$, and which vertices are necessarily on $P$. A feature of a non-trivial block is that, for any three distinct vertices $r, s, t$ in the same block, there exists a path between $r$ and $t$ avoiding $s$, see [3]. On the other hand, if $r$ and $t$ are in different blocks, then all cut-vertices "between" $r$ and $t$ are necessarily on any $r, t$-walk. If we can avoid a vertex, then we can sort of "leap" over that vertex in going from $u$ to $w$. Within a block we can leap to any vertex. In going from one block to another we first leap to the cut-vertex in the first block joining it to the rest of the graph where the other block is located. For any two vertices $u$ and $w$ in different blocks, the leap operation produces the cut-vertex $z$ in the block of $u$ on the way to $w$; in algebraic notation $u+w=z$. If $u$ and $w$ are in the same block, then $u+w=w$.

For the other approach we use the following characterizing property of blocks: for any three distinct vertices $r, s, t$ in the same block, there exists a path between $r$ and $t$ passing through $s$, see [3]. Let $u$ and $w$ be two distinct vertices in the same block, and let $v$ be any other vertex in the same block. Then, in going from $u$ to $w$ we might make a detour along $v$; otherwise stated, we might leap to $v$ first. In algebraic notation: $(u, v, w)$ is a leap. If $u$ and $w$ belong to different blocks, then we may leap to any vertex distinct from $u$ as long as we do not leap over a cut-vertex. In algebraic terms, $(u, v, w)$ is a leap if and only if $u$ and $v$ are two distinct vertices in the same block of $G$, and $u$ is not a cut-vertex between $v$ and $w$ (because otherwise we would leap in the 'wrong direction'). Note that thus the set of leaps is a ternary relation on the vertex set of the graph.

Now the problem is how to translate these approaches in purely algebraic terms, i.e. in terms of operations or relations. We propose sets of axioms for both the operation and the relation. In both cases we define the underlying graph of the 
algebraic structure, and we study the algebraic and the graphic side of this approach.

Throughout this paper $V$ is a finite nonempty set, and $G=(V, E)$ is an undirected, simple graph without loops. The block closure of $G$ is the graph obtained from $G$ by turning every block of $G$ into a complete subgraph. Note that according to the above definition of block a complete graph consists of exactly one block, and in a non-complete graph any bridge with its ends forms a block, whereas all other blocks are 2-connected.

\section{The leap operation}

In this section we introduce the leap operation. The underlying idea of this operation is that it should reflect the following operation on a graph. Let $G=(V, E)$ be a connected graph. If we make a walk from vertex $u$ to vertex $v$, then we have lots of freedom of choice how to walk, but there are certain restrictions. For instance, we have to pass through any cut-vertex lying between $u$ and $v$. If we are only interested in these forced vertices on any walk from $u$ to $v$, then we could skip all other vertices, and just leap from cut-vertex to cut-vertex between $u$ and $v$. So our first "leap" from $u$ carries us to the first cut-vertex $w$ encountered on any $u, v$-path. If there is no such cut-vertex, then we just leap to $v$. We can think of the leap operation as pointing us to this first cut-vertex. Keeping this metaphor in mind, we hope that the axioms for the leap operation are easier to read.

The symbol + denotes a binary operation on the set $V$. The underlying graph $G_{+}=\left(V, E_{+}\right)$of + is defined by

$$
u v \in E_{+} \text {if and only if } u \neq v, u+v=v \text { and } v+u=u .
$$

In the sequel we will consider the following axioms for such a binary operation.

(lo1) $(u+v)+u=u$ for $u, v$ in $V$,

(lo2) if $u \neq v$, then $(u+v)+v \neq u$ for $u, v$ in $V$,

(lo3) if $u+v \neq v$, then $((u+v)+v)+u \neq u$ for $u, v$ in $V$,

(lo4) if $u \neq v=u+v, u+w \neq v$ and $v+w \neq u$, then $u+w=v+w$ for $u, v, w$ in $V$.

In a different context the following basic lemma was proved in [10]. For the sake of completeness, we include its proof here.

Lemma 1 Let + satisfy (lo1) and (lo2). If $u, v \in V$, then

$$
\begin{aligned}
& u+v=u \text { if and only if } u=v, \\
& u+v=v \text { if and only if } v+u=u .
\end{aligned}
$$

Proof. First we prove the second property. If $u+v=v$, then, by axiom (lo 1$)$, we have $v+u=(u+v)+u=u$. Similarly, if $u+v=u$, then we have $u+v=(u+v)+v=v$. 
To prove the first property, let $u, v \in V$. By (lo1), we have $(u+u)+u=u$, so that $((u+u)+u)+u=u+u$. Now with $(u+u)$ playing the role of $u$ and $u$ that of $v$ in (lo2), we have $u+u=u$. Hence, if $u+v=u$, then it follows that $(u+v)+v=u+v=u$, and therefore, by (lo2), we have $u=v$. Conversely, if $u=v$, then it follows that $u+v=u$.

Let + be a binary operation on $V$, and let + satisfy (lo1) and (lo2). Consider $u, v \in V$ such that $u+v \neq v$. Then we have $u \neq v$, for otherwise, by Lemma 1 , we have $u+v=u=v$, which is impossible.

A leap operation on $V$ is a binary operation + satisfying the axioms (lo1), (lo2), (lo3), (lo4). Note that, by Lemma 1 , the underlying graph $G_{+}=\left(V, E_{+}\right)$of a leap operation + can be defined by

$$
u v \in E_{+} \text {if and only if } u \neq v \text { and } u+v=v .
$$

For a connected graph $G=(V, E)$, we define the access operation $+_{G}$ of $G$ by $u+{ }_{G} v=w$, where $w=v$, whenever $u$ and $v$ are in the same block of $G$, and $w$ is the cut-vertex (distinct from $u$ ) in the block of $u$ on any $u, v$-path, whenever $u$ and $v$ are in different blocks of $G$. The next Proposition follows immediately from the definition of the access operation.

Proposition 2 Let $G=(V, E)$ be a connected graph with block closure $B$. Then the access operation $+_{G}$ of $G$ and the access operation $+_{B}$ of $B$ coincide on $V$.

Proposition 3 Let $G=(V, E)$ be a connected graph, and let + be the access operation of $G$. Then + is a leap operation on $V$.

Proof. Let $u, v, w$ be vertices in $G$, and put $x=u+v$. There exists a block $H$ of $G$ containing $u$ and $x$. Obviously if $u \neq x$, then $H$ is determined uniquely.

(lo1): Clearly, $x=u+v$ and $u$ are both in $H$, whence, by definition, we have $u=x+u=(u+v)+u$.

(lo2): If $v$ is in $H$, then $x=v$, and $x+v=v \neq u$, by the definition of the access operation. If $v$ is not in $H$, then $x$ is the cut-vertex in $H$ between $u$ and $v$. Moreover, $x+v$ is not in $H$, so that $(u+v)+v=x+v \neq u$.

(lo3): Now $u+v \neq v$ implies that $u \neq v$, and $v$ is not in $H$ and $x=u+v$ is the cut-vertex in $H$ between $u$ and $v$. Moreover, $y=(u+v)+v$ is not in $H$, so $y+u$ is either $x$ or not in $H$, so that $y+u=((u+v)+v)+u \neq u$.

(lo4): First $u+v=v$ means that $v$ is in $H$. Second $u+w \neq v$ means that $v \neq w$, but also that $v$ is not a cut-vertex between $u$ and $w$. Finally $v+w \neq u$ means that $u$ is not a cut-vertex between $v$ and $w$. Now if $w$ is in $H$, then, by definition, we have $u+w=w=v+w$. So assume that $w$ is not in $H$. Let $z$ be the cut-vertex between $w$ and any vertex in $H$. Then we have $u+w=z=v+w$.

This concludes the proof that the access operation + of $G$ is a leap operation.

Let $u$ and $v$ be any two vertices of a connected graph $G$. By $N_{1}(u, v)$ we denote the set of neighbors $x$ of $u$ with $d(x, v)=d(u, v)-1$, that is, the set of neighbors 
of $u$ one step closer to $v$ than $u$. Recall that a geodetic graph is a connected graph $G$ in which there is a unique shortest $u, v$-path, for any two vertices $u$ and $v$ in $G$. Obviously, every block graph is a geodetic graph.

Theorem 4 Let + be a leap operation on a finite nonempty set $V$, and let $H$ be the underlying graph of + . Then $H$ is a block graph and + is the access operation of $H$.

Proof. Consider an arbitrary component $F$ of $H$.

We first prove that

$$
N_{1}(u, v)=\{u+v\} \text { for all distinct } u, v \in V(F) .
$$

We proceed by induction on $d(u, v)$. Put $n=d(u, v)$. The case $n=1$ follows immediately from the definitions. Let $n>1$. Obviously, $N_{1}(u, v) \neq \emptyset$. Consider an arbitrary $x \in N_{1}(u, v)$. We want to prove that $x=u+v$. Clearly, there exist $u_{0}, u_{1}, \ldots, u_{n} \in V(F)$ such that $u_{0}=u, u_{1}=x, u_{n}=v$ and $u_{0} \rightarrow u_{1} \rightarrow \ldots \rightarrow u_{n}$ is a shortest $u, v$ path in $F$. Suppose, to the contrary, that $u_{1} \neq u_{0}+v$. Since $d\left(u_{1}, v\right)=n-1$, the induction hypothesis implies that $N_{1}\left(u_{1}, v\right)=\left\{u_{1}+v\right\}=\left\{u_{2}\right\}$, and thus $u_{0} \neq u_{1}+v$. By virtue of (lo4) (with $u=u_{0}$, and $u_{1}$ in the role of $v$, and $v$ in the role of $w)$, we conclude that $u_{0}+v=u_{1}+v$. Now $d\left(u_{1}+v, v\right)=n-2$, so that by induction we have $u_{1}+v=u_{2}$, Hence $u_{0}+v=u_{2}$. Thus, by (lo1), we have

$$
u_{2}+u_{0}=\left(u_{0}+v\right)+u_{0}=u_{0} .
$$

By Lemma 1, we have

$$
u_{0}+u_{2}=u_{2}
$$

Hence, by the definition of $H$, the vertices $u_{0}$ and $u_{2}$ are adjacent. Since this would imply that $d\left(u_{0}, v\right)=1+d\left(u_{2}, v\right)=1+n-2=n-1<n$, we get a contradiction. This proves (1).

It follows from (1) that $F$ is a geodetic graph and + restricted to $V(F)$ is the leap operation of $F$.

Next we prove that $F$ is a block graph. Assume the contrary, and let $B$ be a block of $F$ with diameter $k \geq 2$. Let $w$ be a vertex of $B$ having vertices at distance $k$ in $B$. Let $Z$ be the set of vertices in $B$ at distance $k$ from $w$. Since $B$ is a block, there exists a cycle through $w$ and some vertex in $Z$. Let $C$ be such a cycle of minimal length. Going from $w$ along $C$, let $v$ be the first vertex on $C$ in $Z$, let $x$ be the vertex on $C$ just before $v$, and let $u$ be the vertex on $C$ just after $v$. Note that $x$ is not in $Z$, so that we have $d(w, x)=k-1=d(w, v)-1$. Since $F$ is geodetic, $B$ is geodetic too, so we know that $d(w, u) \geq k$. Hence, $k$ being the diameter of $B$, we have $d(w, u)=k$, so that $u$ lies in $Z$ as well. If $u$ were adjacent to $x$, then we could delete $v$ from $C$ obtaining a shorter cycle through $w$ and $Z$. This contradicts the minimality of $C$, so that $u$ and $x$ are not adjacent. Let $y$ be the neighbor of $u$ with $d(w, y)=k-1$. Then we have $u \neq v, v=u+v, u+w=y \neq v$, and $v+w=x \neq u$. So, by (lo4), we must have $y=u+w=v+w=x$, which is a contradiction. This proves that $F$ is a block graph. 
Finally, assume that $H$ is not connected, and let $z$ be a vertex in $V-V(F)$. Choose an arbitrary vertex $w$ in $V(F)$. Then there exists an infinite sequence $\left(w_{0}, w_{1}, w_{2}, \ldots\right)$ such that $w_{0}=w$ and

$$
w_{h+1}=w_{h}+z \text { for each } i \geq 0 .
$$

It follows from $(l o 1)$ that $w_{0} \rightarrow w_{1} \rightarrow \ldots \rightarrow w_{i}$ is a walk in $F$, for each $i \geq 0$. Moreover, it follows from (lo2) that $w_{j} \neq w_{j+2}$ for each $j \geq 0$. Since $V(F)$ is finite, there exist $k$ and $m$, with $0 \leq k<m-2$, such that $w_{k}=w_{m}$ and $w_{k} \rightarrow w_{k+1} \rightarrow$ $\ldots \rightarrow w_{m-1} \rightarrow w_{m}$ is a cycle in $F$. All the vertices on this cycle are in the same block of $F$, so that, $F$ being a block graph, they are mutually adjacent. In particular, we have $w_{k+2}+w_{k}=w_{k}$. Thus $\left(\left(w_{k}+z\right)+z\right)+w_{k}=w_{k}$, which contradicts (lo3). Hence $G$ is connected.

Since $H$ is connected, we have $N_{1}(u, v)=\{u+v\}$, for all distinct $u$ and $v$ in $V$. Moreover, + is the access operation of $H$. This completes the proof.

In mathematical shorthand we could write Theorem 4 as follows: Let + be a leap operation on $V$. Then $G_{+}$is a block graph and $+=+_{G_{+}}$.

Obviously, every block graph is a geodetic graph. A similar result as Theorem 4 was obtained already in Nebeský [9], see also [11]. This result is not as strong as Theorem 4, but on the other hand it concerns all geodetic graphs.

Now we can sharpen Proposition 2 a little bit.

Theorem 5 Let $G=(V, E)$ be a connected graph, and let + be the access operation of $G$. Then + is a leap operation on $V$ and the underlying graph of + is the block closure of $G$.

Proof. First note that, by Proposition 3, the operation + is a leap operation on $V$. Let $B$ be the block closure of $G$. By Proposition 2, the operation + is also the access operation of $B$. Note that, by the definition of the access operation of the block graph $B$, we have $u+v=v$ if and only if $u=v$ or $u$ and $v$ are adjacent in $B$. By the definition of the underlying graph of a leap operation, we conclude that $B$ is the underlying graph of + .

In mathematical shorthand this reads as follows: Let $G$ be a connected graph, then $G_{+_{G}}=B_{G}$.

We recapitulate the above results in a slightly different way, thus obtaining an algebraic approach to block graphs.

Theorem 6 Let + be a binary operation on a finite nonempty set $V$, and let $H$ be the graph of + . Then $H$ is a block graph and + is the access operation of $H$ if and only if + is a leap operation on $V$.

\section{Leap systems}

In this section we introduce another algebraic approach to the block structure of a connected graph $G$. Take any two vertices $u, w$ in $G$. If we can find a $u, w$-path 
passing through a vertex $v$, then, while going from $u$ to $w$, we can first 'leap' to $v$. But we do not allow leaps over vertices that cannot be avoided, that is, we cannot leap over cut-vertices between $u$ and $w$. So what are all the possible leaps in $G$ ? We start at $u$, and going to $w$ we can leap to any vertex in the same block as $u$ (in the 'direction' of $w$ ). If $u$ and $w$ are in the same block, we can also leap from $u$ to $w$. If $u$ and $w$ are not in the same block, then the closest we can get to $w$ is to leap to the cut-vertex $z$ in the block of $u$ that is on a $u, w$-path. Because the structure underlying this algebraic approach is the same as in the case of the leap operation we again use the term leap.

Let $V$ be a finite set, and let $L$ be a ternary relation on $V$. We consider the following axioms for $L$.

$(l s 0)$ if $u \neq v$, then there exists exactly one $y \in V$ with the property that $(u, y, v) \in L$ and $(y, u, v) \notin L \quad(u, v \in V)$;

$(l s 1)$ if $(u, v, w) \in L$, then $(v, u, u) \in L \quad(u, v, w \in V)$;

$(l s 2)$ if $(u, v, w) \in L$, then $u \neq w \quad(u, v, w \in V)$;

$(l s 3)$ if $(u, v, w),(u, w, w) \in L$ and $v \neq w$, then $(v, u, w) \in L \quad(u, v, w \in V)$;

$(l s 4)$ if $(u, v, w),(v, x, w),(x, u, u) \in L$, then $(v, u, w) \in L \quad(u, v, w, x \in V)$;

$(l s 5)$ if $(u, v, v) \in L$ and $u \neq w \neq v$, then $(u, v, w) \in L$ or $(v, u, w) \in L$ $(u, v, w \in V)$;

$(l s 6)$ if $(u, v, w),(v, u, w) \in L$ then there exists $z \in V$ such that $(u, z, w)$, $(v, z, w) \in L$ and $(z, u, w),(z, v, w) \notin L \quad(u, v, w \in V)$.

Let $V$ be a finite nonempty set, and let $L$ be a ternary relation on $V$ satisfying $(l s 0)$. The basic operation of $L$ is the binary operation $+_{L}$ on $V$ defined as follows:

$u+{ }_{L} u=u$, for any $u$ in $V$,

$u+{ }_{L} v$ is the unique element $y$ in $V$ such that $(u, y, v) \in L$ and $(y, u, v) \notin L$, for $u, v$ in $V$ with $u \neq v$.

A leap system on $V$ is a ternary relation $L$ on $V$ satisfying the axioms $(l s 0)-(l s 6)$. By the underlying graph of a leap system $L$ we mean the underlying graph of the basic operation $+_{L}$ of $L$.

Proposition 7 Let $L$ and $M$ be leap systems on a finite nonempty set $V$ with ${ }_{{ }_{L}}=$ ${ }{ }_{M}$. Then $L=M$.

Proof. Assume to the contrary that $L \neq M$. Then there exists $u, v, w$ in $V$ such that $(u, v, w) \in L \backslash M$ or $(u, v, w) \in M \backslash L$. Without loss of generality, let $(u, v, w) \in L \backslash M$. Since $(u, v, w) \in L$, it follows from $(l s 2)$ that $u \neq w$. Let + denote the basic operation of $L$ and $M$. If we would have $(v, u, w) \notin L$, then, by $(l s 0)$, we would have $v=u+w$, so that $(u, v, w) \in M$, which is not the case. Hence $(v, u, w) \in L$. If we would have $(v, u, w) \in M$, then, by $(l s 0)$, we would have $u=v+w$, so that, $(u, v, w)$ being in $L$, we have $(v, u, w) \notin L$, which is impossible. So $(v, u, w) \notin M$. 
Since $(u, v, w),(v, u, w) \in L$, axiom $(l s 6)$ implies the existence of $z$ in $V$ such that $(u, z, w),(v, z, w) \in L$ and $(z, u, w),(z, v, w) \notin L$. From $(l s 0)$ we infer that this element $z$ is unique. Moreover, the definition of the basic operation of $L$ tells us that $z=u+w=v+w$.

Now + is also the basic operation of $M$. So $(u, z, w),(v, z, w) \in M$. By $(l s 2)$, we have $u \neq w \neq v$. Since $(u, v, w) \in L$, it follows from $(l s 1)$ that $(u, v, v) \in L$. By $(l s 2)$, we have $u \neq v$, and therefore $(v, u, v) \notin L$. Combining $(l s 0)$ with the definition of + , we get $u+v=v$. Since + is also the basic operation of $M$, we have $(u, v, v) \in M$. Since $u \neq w \neq v$, it follows from $(l s 5)$ that $(u, v, w) \in M$ or $(v, u, w) \in M$. Since this is impossible, we have a contradiction, which settles the proof.

Theorem 8 Let $L$ be a leap system on a finite nonempty set $V$, and let + be the basic operation of $L$. Then + is a leap operation on $V$.

Proof. In the sequel let $u$ and $v$ be elements in $V$ with $u \neq v$. By $(l s 0)$, there exists a unique element $y$ in $V$ such that $(u, y, v) \in L$ and

$$
(y, u, v) \notin L .
$$

So, by definition, we have $y=u+v$.

We check the four axioms of a leap operation.

(lo1): First we have $(u+u)+u=u+u=u$, by definition. So the case $u \neq v$ remains. By $(l s 1)$, we have $(y, u, u) \in L$. Hence, by $(l s 2)$, we have $y \neq u$. Now by $(l s 0)$, we deduce the existence of a unique $t$ in $V$ such that $(y, t, u) \in L$ and $(t, y, u) \notin L$. Hence $t=y+u$. Suppose that $t \neq u$. Since $(y, u, u),(y, t, u) \in L$, axiom $(l s 3)$ would imply that $(t, y, u) \in L$, which is a contradiction. Hence it follows that $t=u$ and the verification of (lo1) is complete.

(lo2): If $y=v$, then $(u+v)+v=y+y=y=v \neq u$. So assume that $y \neq v$. Then, by $(l s 0)$, there exists a unique $x$ in $V$ such that $(y, x, v) \in L$ and $(y, x, v) \notin L$. Hence

$$
x=y+v=(u+v)+v .
$$

If $x=u$, then $(y, u, v) \in L$, which contradicts (2). Hence $x \neq u$, by which we have verified (lo2).

(lo3): Let $u+v \neq v$. Then $u \neq v$. We have $y \neq v$. Let $x$ be divined as in the verification of $(l o 2)$. Then $(y, x, v) \in L$. Assume to the contrary that $u=$ $((u+v)+v)+u=x+u$. Hence $u=x+u$. Then, by definition, we have $(x, u, u) \in L$. Recall that $(u, y, v) \in L$. Since $(y, x, v) \in L$, it follows that (ls4) implies that $(y, u, v) \in L$, which contradicts (2). Thus the verification of (lo3) is complete.

(lo4): Let $u+v=v, u+w \neq v$ and $v+w \neq u$. Then we have $v \neq w$. Since $u+v=v$, Lemma 1 implies that $v+u=u$. This implies that $w \neq u$. By (ls5), we have $(u, v, w) \in L$ or $(v, u, w) \in L$. Without loss of generality, let $(u, v, w) \in L$. If $(v, u, w) \notin L$, then $v=u+w$; a contradiction. Thus $(v, u, w) \in L$. By $(l s 6)$, there exists $z \in V$ such that $(u, z, w),(v, z, w) \in L$ and $(z, u, w),(z, v, w) \notin L$. By virtue of (lso), $u+w=z=v+w$, which completes the verification of (lo4). 
Let $G=(V, E)$ be a connected graph. Then, for $u, v, w$ in $V$, the triple $(u, v, w)$ is a leap in $G$ if $v$ belongs to a $u, w$-path in $G$, and $u \neq v$, and there is no cut-vertex in $G$ separating $u$ and $v$. Note that, in a leap $(u, v, w)$, the vertices $u$ and $v$ belong to the same block. Moreover if $u, v, w$ are in the same block with $u$ distinct from $v$ and $w$, then $(u, v, w)$ is always a leap in $G$. Clearly, the set $L_{G}$ of all leaps in $G$ is a ternary relation on $V$. The following lemma is an easy consequence of the definition and of these observations.

Lemma 9 Let $G$ be a connected graph with block closure B. Then the set of leaps $L_{G}$ in $G$ is identical with the set of leaps $L_{B}$ in $B$.

In mathematical shorthand this reads as follows: Let $G$ be a connected graph. Then $L_{G}=L_{B_{G}}$.

There is a close connection between the set of leaps of a connected graph $G$ and the all path transit function of $G$, which was characterized by Changat, Klavžar and Mulder [1]. This connection is similar to the connection between the interval function of $G$ (widely studied in Mulder [5] and characterized in Nebeský [7]) and the set of all steps in $G$ (introduced and characterized in Nebeský [8]). Note that the all-paths transit function and the interval function are important instances of transit functions in the sense of Mulder [6]; another instance of a transit function was studied by Morgana and Mulder [4].

Theorem 10 Let $G=(V, E)$ be a connected graph, and let $L$ be the set of leaps in $G$. Then $L$ is a leap system on $V$.

Proof. By Lemma 9, we may assume without loss of generality that $G$ is a block graph. This means that, if $r \neq s$ and $(r, s, t)$ is a leap in $G$, then $s$ is a neighbor of $r$ on some $r, t$-path. From this observation we deduce immediately axioms $(l s 0),(l s 1)$, and $(l s 2)$.

$(l s 3)$ : Let $(u, v, w),(u, w, w) \in L$ and $v \neq w$. Then $u$ is adjacent to $v$ as well as $w$, so $v \rightarrow u \rightarrow w$ is a path in $G$. Hence $(v, u, w)$ is a leap in $G$.

$(l s 4)$ : Let $(u, v, w),(v, x, w),(x, u, u) \in L$. Then $u v, v x, v u \in E$. Therefore $u, v, x$ induce a triangle in $G$, and hence are in the same block, say $S$. Since $(v, x, w) \in L$, we have $v \neq w$. If $w$ lies in $S$, the we have $(v, u, w) \in L$. Assume that $w$ does not belong to $S$. Since $(v, x, w) \in L$, it follows that $v$ is not a cut-vertex between $x$ and $w$. Now, since $u, v, x$ induce a triangle, it follows again that $(v, u, w) \in L$.

$(l s 5)$ : Let $(u, v, v)$ be a leap in $G$, so that $u$ and $v$ are adjacent, and let $w$ be a vertex distinct from $u$ and $v$. If $w$ is in the same block as $u$ and $v$, then both $(u, v, w)$ and $(v, u, w)$ are leaps in $G$. So assume that $w$ is not in the same block. If $u$ is not a cut-vertex between $v$ and $w$, then there exists a path between $u$ and $w$ with $v$ as vertex second vertex on that path. Hence $(u, v, w)$ is a leap. Otherwise, if $u$ is a cut-vertex between $v$ and $w$, then, by definition, $(v, u, w)$ is a leap.

$(l s 6)$ : Let $(u, v, w)$ and $(v, u, w)$ be leaps in $G$. Then $u$ and $v$ are adjacent and $u \neq w \neq v$. Let $F$ be the block of $G$ containing $u$ and $v$. Note that $F$ is complete, $G$ being a block graph. If $w$ does not belong to $F$, then there is a cut-vertex $z$ in $F$ such that $w$ and the edge $u v$ belong to different components of $G-z$. If $w$ 
belongs to $F$, then we set $z=w$. In both cases we have $(u, z, w),(v, z, w) \in L$ and $(z, u, w),(z, v, w) \notin L$.

Proposition 11 Let $G=(V, E)$ be a connected graph, and let $L$ be the set of leaps in $G$. Then the basic operation $+_{L}$ of $L$ and the access operation $+_{G}$ of $G$ are identical.

Proof. We have to prove that $u+_{L} v=u+_{G} v$, for all $u$ and $v$ in $V$. By Theorem 10, $L$ satisfies axioms $(l s 0)-(l s 6)$.

Choose any two vertices $u$ and $v$ in $V$. If $u=v$, then, by definition, we have $u+{ }_{L} v=u+{ }_{L} u=u$ as well as $u+{ }_{G} v=u+{ }_{G} u=u$.

So assume that $u \neq v$. By the definition of the basic operation $+_{L}$ of $L$, the element $u+{ }_{L} v$ is the unique vertex $x$ in $G$ such that $(u, x, v)$ is a jump in $G$ and $(x, u, v)$ is not. First let $u$ and $v$ be in the same block $H$ of $G$. Then $(u, v, v)$ is a jump and $(v, u, v)$ is not, so $x=v$. On the other hand $u+_{G} v=v$, by definition of the access operation of a graph. Now let $u$ and $v$ be vertices that are not in the same block of $G$, and let $x$ be the cut-vertex in the same block as $u$ separating $u$ and $v$. Then, by the definition of $+_{G}$, we have $u+_{G} v=x$. Moreover, by the definition of $L$, we have $(u, x, v)$ in $L$ but $(x, u, v)$ not in $L$. So, by virtue of axiom $(l s 0)$, we have $u+{ }_{L} v=x$, which completes the proof.

In mathematical shorthand this reads as follows: Let $G=(V, E)$ be a connected graph. Then $+_{L_{G}}=+_{G}$.

We conclude this paper by restating the above results in a series of theorems and corollaries, so as to have a different perspective on these results.

Theorem 12 Let $L$ be a leap system on a finite nonempty set $V$, and let $H$ be the underlying graph of $L$. Then $H$ is a block graph and $L$ is the set of leaps of $H$.

Proof. Let + denote the basic operation of $L$. By definition, $H$ is the underlying graph of + . By Theorem 8, the operation + is a leap operation on $V$. From Theorem 4 we infer that $H$ is a block graph and that + is the access operation of $H$. Let $M$ denote the set of leaps in $H$. By Theorem 10, $M$ is a leap system on $V$. By Proposition 11, + is the basic operation of $M$. From Proposition 7 we infer that $L=M$, which concludes the proof.

In mathematical shorthand this reads as follows: Let $L$ be a leap system. Then $L_{G_{L}}=L$.

Theorem 13 Let $G$ be a connected graph with set of leaps $L$. Then the underlying graph $G_{L}$ of $L$ and block closure $B$ of $G$ are identical.

In mathematical shorthand this reads as follows: Let $G$ be a connected graph. Then $G_{L_{G}}=B_{G}$.

We recapitulate the above results in a slightly different way, thus obtaining another algebraic approach to block graphs. 
Theorem 14 Let $L$ be a ternary relation on a finite nonempty set $V$ with underlying graph $H$. Then $H$ is a block graph with set of leaps $L$ if and only if $L$ is a leap system on $V$.

Finally, we restate Theorems 6 and 14 as a characterization of block graphs in terms of leap operations and leap systems.

Theorem 15 Let $G=(V, E)$ be a graph. Then the following statements are equivalent:

(a) $G$ is a block graph,

(b) $G$ is the underlying graph of a leap operation on $V$,

(c) $G$ is the underlying graph of a leap system on $V$.

\section{References}

[1] M. Changat, S. Klavžar, H.M. Mulder: The all path transit function of a graph. Czecoslovak Math. Journal 51 (2001) 439 - 448.

[2] F. Harary: A characterization of block graphs. Canad. Math. Bull. 6 (1963) 1 6.

[3] F. Harary, Graph Theory, Addison-Wesley, Reading MA, 1969.

[4] M.A. Morgana, H.M. Mulder: The induced path convexity, betweenness, and svelte graphs. Discrete Math. 254 (2002) 349 - 370.

[5] H.M. Mulder: The interval function of a graph. MC-tract 132, Mathematish Centrum. Amsterdam 1980.

[6] H.M. Mulder: Transit functions on graphs. In preparation.

[7] L. Nebeský: A characterization of interval function of a graph. Czechoslovak Math. Journal 44 (119) 1994, 173 - 178.

[8] L. Nebeský: Geodesics and steps in a connected graph. Czechoslovak Math. Journal 47 (122) 1997, 149 - 161.

[9] L. Nebeský: An algebraic characterization of geodetic graphs. Czechoslovak Math. Journal 48 (123) 1998, 707 - 709.

[10] L. Nebeský: A tree as a finite nonempty set with a binary operation. Mathematica Bohemica 125 (2000) 455 - 458.

[11] L. Nebeský: New proof of a characterization of geodetic graphs. Czechoslovak Math. Journal 52 (127) 2002, 33 - 39. 